\title{
Arteriovenous Fistulae and Steal Syndrome Complicating Renal Allograft Biopsies. A Report of Two Cases
}

\author{
Ramessur Chandran Sharmila ${ }^{1 *}$, Mulley William ${ }^{1,2}$, Holt Michael ${ }^{3}$, Burnes James ${ }^{3}$ and Kanellis John ${ }^{1,2}$ \\ ${ }^{1}$ Department of Nephrology, Monash Medical Centre, Clayton, Australia \\ ${ }^{2}$ Department of Medicine, Monash University, Clayton, Australia \\ ${ }^{3}$ Southern Health Diagnostic Imaging, Monash Medical Centre, Clayton, Australia
}

\begin{abstract}
Background: Biopsy-related kidney transplants Arteriovenous Fistulae (AVF) are common. Most resolve spontaneously but rarely are they complicated by graft dysfunction. We describe 2 such cases of AVF occurring post renal allograft biopsy.

Case reports: A 33 year old lady had multiple biopsies of her renal allograft due to ongoing rejection. Her renal function worsened and a $1.3 \mathrm{~cm}$ AVF was diagnosed on Doppler Ultrasound. The AVF closed spontaneously during angiogram and the patient's renal function improved.

The second case was a 25 year old man who had a biopsy due to acute rise in creatinine 14 weeks post transplantation. The biopsy was complicated by haematuria and an AVF was diagnosed on doppler ultrasound. The AVF enlarged over 3 months. An angiogram and endovascular coiling was performed with good resolution of the AVF. The patient's renal function normalised.
\end{abstract}

Conclusion: Endovascular coiling can ensure good resolution of renal AVF and is generally well tolerated.

Keywords: Arteriovenous fistula; Endovascular coiling; Percutaneous biopsy; Renal transplant

Abbreviations: AVF: Arteriovenous Fistulae; HLA: Human Leucocyte Antigen

\section{Background}

Biopsy-related kidney transplant Arteriovenous Fistulae (AVF) are reported to be common (5-15\%) [1,2]. The majority remains undiagnosed and resolve spontaneously. However in a small number of cases, they can be complicated by significant graft dysfunction, haematuria, refractory hypertension and steal syndrome [2]. We describe the course and outcome of two complicated cases of AVF post biopsy.

\section{Case Reports}

Between January 2005 and January 2010, 915 renal transplant biopsies were performed at the Monash Medical Centre, under direct ultrasound guidance using a 15 gauge direct cutting biopsy needle after local anesthesia is infiltrated. Two patients developed renal transplant AVF associated with significant graft dysfunction. The 2 biopsies were performed by 2 different nephrology registrars. No other cases were identified however patients were not routinely monitored for this complication. In both cases, basiliximab was given at induction together with tacrolimus, mycophenolate mofetil and prednisolone. The last 3 drugs were continued as maintenance immunosuppression.

The first case was a 33 year old lady with end stage renal failure secondary to interstitial nephritis, who developed early antibodymediated rejection in her third renal graft, requiring multiple biopsies. The graft was from a 57 year old cadaveric brain dead donor with 5/6 HLA (Human Leucocyte Antigen) mismatches. There was no drop in haemoglobin or haemodynamic compromise during any of the biopsies. The patient's clotting profile done prior to each biopsy, remained normal. Eleven months post-transplant, her creatinine rouse acutely from $103 \mu \mathrm{mol} / \mathrm{L}$ to $170 \mathrm{umol} / \mathrm{L}$. A $1.3 \mathrm{~cm}$ AVF was diagnosed on colour doppler ultrasound and coiling was planned. During the formal angiogram, the fistula closed spontaneously after the initial flush of contrast, likely secondary to spontaneous arterial spasm. Creatinine fell promptly to $126 \mu \mathrm{mol} / \mathrm{L}$ and remained $89 \mu \mathrm{mol} / \mathrm{L} 24$ months later.
Case two was a 25 year old man with end stage renal failure secondary to reflux nephropathy who received a 2/6 HLA mismatched graft from his 60 years old father. Fourteen weeks post-transplant, his creatinine rouse from $172 \mu \mathrm{mol} / \mathrm{L}$ to $228 \mu \mathrm{mol} / \mathrm{L}$. A renal biopsy was performed under direct ultrasound guidance. Two passes were made in the inferior pole. Three hours post procedure, the patient experienced macroscopic haematuria, which spontaneously resolved after 24 hours. He remained haemodynamically stable during that time and his haemoglobulin and clotting profile remained in the normal range. A colour doppler ultrasound revealed a $1.3 \mathrm{~cm}$ inferior pole fistula (Figure 1). Histopathology showed only mild donor-related nephrosclerosis. Over the ensuing 3 months, fistula size remained unchanged but the creatinine gradually rouse to $324 \mu \mathrm{mol} / \mathrm{l}$, prompting intervention. An angiogram showed the fistula, complicated by steal with poor peripheral perfusion of the graft (Figure 2A). Coiling was performed with seven $2-5 \mathrm{~mm}$ vortex-fibre coils. The fistula closed with good return of perfusion to the renal parenchyma (Figure 2B). Creatinine fell rapidly to $167 \mu \mathrm{mol} / \mathrm{L}$ and remained stable 30 months later.

\section{Discussion}

Renal biopsy remains the gold standard investigation for suspected intrinsic renal disease and generally carries a very low risk. However previous reports suggest that in up to $15 \%$ of cases [1-3], an AVF can occur as a consequence of simultaneous damage to the arterial and venous blood vessel walls and subsequent aberrant repair process [2]. The AVF occasionally forms weeks to months after injury [2]. In most cases, the AVF remains asymptomatic and resolves spontaneously

*Corresponding author: Ramessur Chandran Sharmila, Department of Nephrology, Monash Medical Centre, Clayton, Australia, E-mail: sharmila.ramessur@monash.edu

Received August 31, 2012; Accepted September 17, 2012; Published September 19, 2012

Citation: Sharmila RC, William M, Michael H, James B, John K (2012) Arteriovenous Fistulae and Steal Syndrome Complicating Renal Allograft Biopsies. A Report of Two Cases. J Clin Case Rep 2:202. doi:10.4172/2165-7920.1000202

Copyright: (c) 2012 Sharmila RC, et al. This is an open-access article distributed under the terms of the Creative Commons Attribution License, which permits unrestricted use, distribution, and reproduction in any medium, provided the original author and source are credited. 
Citation: Sharmila RC, William M, Michael H, James B, John K (2012) Arteriovenous Fistulae and Steal Syndrome Complicating Renal Allograft Biopsies. A Report of Two Cases. J Clin Case Rep 2:202. doi:10.4172/2165-7920.1000202

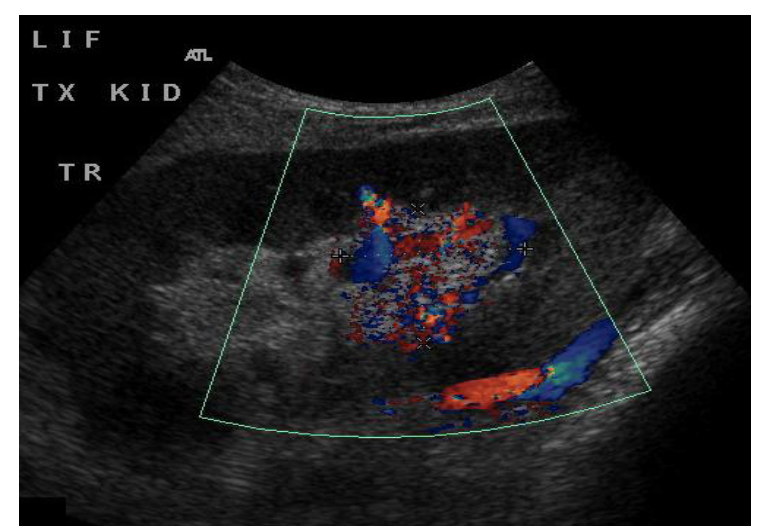

Figure 1: Colour doppler ultrasound of the renal allograft showing AVF due to percutaneous needle biopsy.

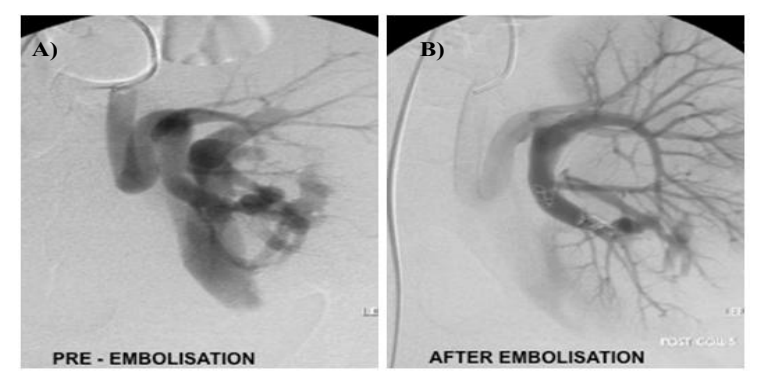

Figure 2: A) Selective angiogram showing inferior pole AVF complicated by steal with poor peripheral perfusion. B) Angiogram after embolisation with seven 2-5 mm GDC18 Vortex-fibre coils: occlusion of the AVF and improvement in renal perfusion.

$[1,3,4]$, but in approximately $20 \%$ of cases, complications can occur, including persistent haematuria, refractory hypertension, worsening renal function, steal syndrome and rupture $[2,5]$.

Risk factors for developing renal biopsy related AVF include transplanted, as compared to native kidneys [6], older grafts, multiple biopsies and several passes during a biopsy [5]. Endovascular coiling is currently the most effective and safest method of closure of AVF [5-8]. It is performed by catheterisation of the artery feeding the AVF and insertion of platinum microcoils $[3,7,9]$. It is well tolerated with bleeding and renal infarction reported as complications in only minority of cases [5-7].

These cases highlight the need to consider a biopsy-related AVF in renal transplant recipients where graft dysfunction develops or further progresses after a biopsy is performed. Prompt imaging can identify the AVF, which should be monitored for an increase in size by doppler ultrasound, every 3 to 6 months [10,11]. Endovascular coiling is recommended if there is expansion of the AVF on ultrasound doppler examination or if graft dysfunction occurs with no other obvious cause [10].

\section{Acknowledgements}

Renal Department of the Monash Medical Centre, Victoria, Australia.

Radiology Department of the Monash Medical Centre, Victoria, Australia.

\section{Conflicts of Interest}

None to declare for all authors. The results presented in this paper have not been published previously in whole or part, except in abstract format.

\section{References}

1. Iloreta AT, Blaufox MD (1979) Natural history of post-biopsy renal arteriovenous fistula: a 10-year follow-up. Nephron 24: 250-253.

2. Nakatani T, Uchida J, Han YS, Iwai T, Nakamura K, et al. (2003) Renal allograft arteriovenous fistula and large pseudoaneurysm. Clin Transplant 17: 9-12.

3. Rüth EM, Dittrich K, Jüngert J, Uder M, Rascher W, et al. (2008) Successful interventional treatment of arteriovenous fistula after kidney biopsy in pediatric patients--a report of three cases. Nephrol Dial Transplant 23: 3215-3218.

4. Shaheen F, Hakeem A, Singh M, Gojwari T, Shafi H, et al. (2008) Color Doppler findings of post-biopsy arteriovenous fistula in renal transplant. Indian J Nephrol 18: 132-133

5. Loffroy R, Guiu B, Lambert A, Mousson C, Tanter Y, et al. (2008) Management of post-biopsy renal allograft arteriovenous fistulas with selective arterial embolization: immediate and long-term outcomes. Clin Radiol 63: 657-665.

6. Bilge I, Rozanes I, Acunas B, Minareci O, Nayir A, et al. (1999) Endovascular treatment of arteriovenous fistulas complicating percutaneous renal biopsy in three paediatric cases. Nephrol Dial Transplant 14: 2726-2730.

7. Lorenzen J, Schneider A, Körner K, Regier M, Adam G, et al. (2012) Postbiopsy arteriovenous fistula in transplant kidney: treatment with superselective transcatheter embolisation. Eur J Radiol 81: e721-e726.

8. Kopecna L, Mach V, Prochazka J (2005) Arteriovenous fistula as a complication of renal biopsy. Bratisl Lek Listy 106: 218-220.

9. Bozgeyik Z, Ozdemir H, Orhan I, Cihangiroglu M, Cetinkaya Z (2008) Pseudoaneurysm and renal arteriovenous fistula after nephrectomy: two cases treated by transcatheter coil embolization. Emerg Radiol 15: 119-122.

10. Hübsch P, Schurawitzki H, Traindl O, Karnel F (1991) Renal allograf arteriovenous fistula due to needle biopsy with late onset of symptoms-diagnosis and treatment. Nephron 59: 482-485.

11. Manganelli R, lannaccone S, De Simone W (2011) Role of the ultrasounds in the kidney biopsy: a case of postbiopsy hematoma linked to renal arteriovenous fistula. G Ital Nefrol 28: 323-328. 